"The submitted manuscript has been authored by a contractor of the U.S. Government under contract No. DE-AC05-84OR21400. Accordingly, the U.S

Government retains a nonexclusive royalty-free

license to publish or reproduce the published form of

this contribution, or allow others to do so, for U.S.

Government purposes.'

\title{
Modeling of plume dynamics in laser ablation processes for thin
} film deposition of materials*

J. N. Leboeuf ${ }^{\dagger}$, K. R. Chen ${ }^{\text {a) }}$, J. M. Donato, D. B. Geohegan, C. L. Liu, A. A. Puretzkyb), and R. F. Wood

Oak Ridge National Laboratory, P. O. Box 2009, Oak Ridge, Tennessee 37831-8071

\begin{abstract}
The transport dynamics of laser-ablated neutral/plasma plumes are of significant interest for film growth by pulsed-laser deposition of materials since the magnitude and kinetic energy of the species arriving at the deposition substrate are key processing parameters. Dynamical calculations of plume propagation in vacuum and in background gas have been performed using particle-in-cell hydrodynamics, continuum gas dynamics, and scattering models. Results from these calculations are presented and compared with experimental observations.
\end{abstract}

[PACS: 52.65, 81, 52.50.J, 52.75]

* Paper 1IA.04, Bull. Am. Phys. Soc. 40, 1644 (1995).

$\dagger$ Invited speaker.

a) Permanent address: Department of Physics, National ChangHua University of Education, ChangHua, Taiwan.

b) Permanent address: Institute of Spectroscopy, Russian Academy of Sciences, Troitsk, Russia. 


\section{INTRODUCTION}

Pulsed-laser deposition (PLD) offers an efficient and versatile process to grow high quality films of various materials, including high-temperature superconducting thin films. ${ }^{1}$ In this process, a pulsed laser, usually of the excimer variety such as $\mathrm{KrF}$ (krypton fluoride) and $\mathrm{ArF}$ (argon fluoride) with pulse lengths of tens of nanoseconds, is used to ablate material gently at fluences of a few $\mathrm{J} / \mathrm{cm}^{2}$. The resulting neutral/weakly ionized plasma plume is allowed to expand, in vacuum or in background gas, before depositing on a substrate of compatible material at a suitable distance away from the target.

While laser ablation is reportedly simple conceptually and experimentally, ${ }^{2}$ the physics ingredients that come into play are quite complicated ${ }^{3}$ given that they involve lasersolid interactions at the target, plasma formation off the target, vapor/plasma plume transport toward the deposition substrate with its associated hydrodynamics and atomic physics, as well as plume-solid interactions at the deposition substrate.

We have been pursuing a global physics and computational modeling approach to laser ablation that relies on thermal models to describe laser-solid interactions for neutral plume formation; on kinetic breakdown models of plasma formation in the vapor plume; on variety of hydrodynamics, gas dynamics, and collisional or scattering transport models for the neutral/weakly ionized plasma plume; as well as on molecular dynamics methods to treat plume-substrate interactions. The many facets of this modeling effort have been summarized elsewhere. ${ }^{4}$ Here we concentrate on the dynamics of plume propagation and on comparisons with experimental results.

Experimental observations have shown marked differences between plume expansion in vacuum and in the presence of a higher pressure background gas. These observations are common to a wide range of laser-ablated materials including silicon, carbon, yttrium, and high-temperature superconducting compounds such as YBCO (yttrium-barium-copper oxide). Ablation in high-pressure ambient gases results in shock 
waves and expansion fronts propagating through the background gases. ${ }^{5}$ Time-of-flight measurements also show two components in the ion probe signals: an energetic component that propagates at vacuum speed and another that is more or less significantly slowed down depending on the pressure of the background gas. 6 These differences are important since the magnitude and kinetic energy of the species arriving at the deposition substrate are key processing parameters.

We have applied our transport models to study plume expansion in near vacuum and in a higher pressure background gas. Results from some of these models will be described starting from more qualitative but higher dimensional ones and proceeding to the more detailed and flexible one-dimensional (1-D) ones. Section II contains results from particle-in-cell hydrodynamics models, while Sec. III pertains to gas dynamics ones. Section IV is devoted to collisional or scattering models. A summary is given in Sec. V.

\section{PARTICLE-IN-CELL HYDRODYNAMICS MODELS OF PLUME TRANSPORT}

A qualitative exploration of plume expansion in vacuum and in background gas has been carried out using a versatile two-dimensional (2-D) particle-in-cell hydrodynamics model. ${ }^{7,8}$ In this model, many particles are used to represent elements or cells of the fluid and are followed in space and time in their pressure gradient field. The trajectories of each individual particle or fluid element $\mathrm{i}$ are traced in space and time according to the following equations of motion for positions $\vec{x}_{1}$ and velocities $\vec{v}_{i}$ :

$$
\begin{aligned}
& \frac{d \vec{x}_{i}}{d t}=\vec{v}_{i}, \\
& \frac{d \vec{v}_{i}}{d t}=-\left.\frac{1}{M} \frac{\vec{\nabla} P}{n}\right|_{\vec{x}=\vec{x}_{i}}-v\left(\vec{v}_{i}-\left.V_{f}\right|_{\bar{x}=\vec{x}_{i}}\right),
\end{aligned}
$$


where $\mathrm{M}$ is the mass of the particle. The second term on the right-hand side of Eq. (lb) is a viscous drag term with coefficient $v$. Setting $v=1$ means that all particles in a given cell are constrained to the average velocity of all particles in that particular cell, while setting $\mathrm{v}=$ 0 means that particles are allowed to stream freely even within one cell. The density $\mathrm{n}$ and fluid velocity $\mathrm{V}_{\mathrm{f}}$ are accumulated on an auxiliary grid from the positions and velocities of the particles according to

$$
n(\vec{x})=\sum_{i} S\left(\vec{x}-\vec{x}_{i}\right),
$$

$$
V_{f}(\vec{x})=\frac{\sum_{i} S\left(\vec{x}-\vec{x}_{i}\right) \vec{v}_{i}}{\sum_{i} S\left(\vec{x}-\vec{x}_{i}\right)},
$$

where the sum is over all particles and the quantity $S\left(\vec{x}-\vec{x}_{i}\right)$ represents the distribution of mass about the central point $\vec{x}_{i}$ of the particle. An isentropic equation of state is assumed, and the total pressure $\mathrm{P}$ is expressed as $\mathrm{P}=$ const $\times \mathrm{n}^{\gamma}$, with $\gamma=2$. These equations are solved using finite differences in space and the standard leapfrog scheme ${ }^{9}$ for integration in time of Eq. (1). More details on the numerics can be found in Refs. 7 and 8.

For the results that follow, a two-dimensional Cartesian representation is used with a system size of 64 grid points in both $\mathrm{x}$ and $\mathrm{y}$ directions. The plume is represented by a high-density, sharp boundary pillbox with eight grid points on each side (Fig. 1), which is then allowed to expand under its own pressure. The background gas, when present, is made up of much fewer particles distributed uniformly over the whole area. In the example shown in Fig. 1, the background is 256 times more dilute than the initial plume, which is represented with 1024 particles per cell and $v=0$. Comparing the top (plume in vacuum) to the middle set of frames (plume and background together) with time going from left to right, it is clear that the plume expansion is slowed down by the background gas. The bottom frames that display the time evolution of the background particles alone show that the background gas is extruded and pushed ahead by the much higher density plume. A 
mixing zone between plume and background particles, which corresponds to the region where background particles pile up and the density gradient is reversed thus slowing down and even stopping some of the plume particles, is apparent in the middle frames of Fig. 1. We also note that many plume particles have been able to tunnel through the background gas in this free streaming model.

Another interesting feature of the particle-in-cell model is that the character of the dynamics can be changed by setting the drag coefficient $v$ to $0.0,0.01$, and 1.0 . We have displayed the $\mathrm{x}-\mathrm{y}$ space projection of plume and background particles after 400 time steps in the calculation for these different values of $v$ in Fig. 2. It is clear from this figure that the interaction between plume and background particles becomes more and more collisional as $v$ is increased. The whole plume is considerably slowed down in the process in a manner similar to having the plume interact with an increasingly higher pressure background gas. 5

\section{GAS DYNAMICS MODELS OF PLUME TRANSPORT}

\section{A. 2-D model and results}

To investigate the dynamic features of plume transport in vacuum and in background gas, we have also used a conventional 2-D gas dynamics model that solves conservation equations for mass density $(\rho)$, momentum $(\rho \vec{v})$, and energy $(\rho e+1 / 2 \rho v 2)$ :

$$
\frac{\partial}{\partial \mathrm{t}}\left\{\begin{array}{l}
\rho \\
\rho \overrightarrow{\mathrm{v}} \\
\rho \mathrm{e}+\frac{1}{2} \rho \mathrm{v}^{2}
\end{array}\right\}=-\vec{\nabla} \cdot\left\{\begin{array}{l}
\rho \overrightarrow{\mathrm{v}} \\
\mathrm{P} \overrightarrow{\mathrm{I}}+\rho \overrightarrow{\mathrm{v}} \overrightarrow{\mathrm{v}} \\
\overrightarrow{\mathrm{v}}\left[\left(\rho \mathrm{e}+\frac{1}{2} \rho \mathrm{v}^{2}\right)+\mathrm{P}\right]
\end{array}\right\} .
$$

They are augmented by the following equation of state for internal energy e and pressure P:

$$
\mathrm{P}=(\gamma-1) \rho \mathrm{e}=\frac{\rho}{\mathrm{M}} \mathrm{k}_{\mathrm{B}} \mathrm{T},
$$

with $\gamma=5 / 3$. 
This model is numerically implemented using finite differences in Cartesian space and the Rusanov scheme ${ }^{9}$ in time. This simple explicit implementation allows the background and plume densities to be monitored independently through their respective mass conservation equations, albeit with the same advection velocity. In addition, the total density advected using Eq. 3 is identical to the sum of its background and plume parts. The system size in the $\mathrm{x}$ and $\mathrm{y}$ dimensions is chosen to be $100 \times 100$ grid points, which nominally cover $5 \mathrm{~cm}$ in each direction. The plume is represented by a Gaussian in density and temperature centered halfway in the $y$ direction and at $x=0$ with a half-width of 4 grid points in each direction. The density and temperature maxima are set at $10^{19} \mathrm{~cm}^{-3}$ and $7000 \mathrm{~K}$, respectively. The background, when present, is uniform in density and at room temperature $(293 \mathrm{~K})$ over the entire domain. Both plume and background are stationary at time $\mathrm{t}=0$.

Results from this 2-D model are displayed in Fig. 3 for plume expansion in near vacuum (ratio of plume to background densities $=10^{-11}$; top) and in background gas at a pressure of $\sim 1$ Torr (bottom). Contours of total density (plume plus background) are shown as time progresses from left to right. It is evident that strong shocks are generated as the plume expands in presence of the background gas. The plume does, in fact, snowplow the background gas, giving rise to the crescent feature at the leading edge, which is clearly seen in Fig. 3. There is virtually no mixing between plume and background in this highly collisional gas dynamics model. We note that the gross characteristics of the expansion in near vacuum and in background gas are remarkably similar to the light emission patterns experimentally detected using a gated Intensified Charge-Coupled Device (ICCD) camera for laser ablation of yttrium in argon at a pressure of 200 mTorr with a KrF laser. 6

One of the technical issues for PLD is the capability of attaining large area deposition of uniform thin films. Many approaches have been tried to improve uniformity. ${ }^{2}$ We have explored the idea of using two overlapping plumes, produced with 
two lasers or a single laser with a split beam, as a way to obtain large area deposition and better uniformity. We have modeled expansion and transport of these two interacting plumes in near vacuum using the 2-D gas dynamics model; a typical output is shown in Fig. 4. The two plumes are initialized like the single plume of Fig. 3 with a separation of 50 grid points between their centers on a $200 \times 200$ grid in the $\mathrm{x}$ and $\mathrm{y}$ directions. As time goes by, both plumes expand until they enter in contact with each other and a shock forms. A much smoother propagating front covering a large area has developed as a result of the interaction.

\section{B. 1-D model and results}

Characteristics of plume expansion in vacuum and in background gas have been examined more quantitatively using a 1-D gas dynamic model that solves the same conservation equations as the 2-D model but includes plasma effects such as ionization through the Saha equation and energy input through laser light absorption. The set of equations used in the 1-D gas dynamics model is as follows:

$$
\frac{\partial}{\partial t}\left\{\begin{array}{l}
\rho \\
\rho v_{x} \\
\rho e+\frac{1}{2} \rho v_{x}^{2}
\end{array}\right\}=-\frac{\partial}{\partial x}\left\{\begin{array}{l}
\rho v_{x} \\
\rho v_{x}^{2}+P-\mu \frac{\partial v_{x}}{\partial x} \\
v_{x}\left[\left(\rho e+\frac{1}{2} \rho v_{x}^{2}\right)+P-\mu \frac{\partial v_{x}}{\partial x}\right]
\end{array}\right\}+\left\{\begin{array}{l}
S_{p} \\
0 \\
\alpha \Phi(t) e^{-\alpha x}+S_{e}
\end{array}\right\}
$$

with $\mu$ denoting the viscosity, $\alpha$ the light absorption coefficient, $\Phi$ the laser energy input, and $\mathrm{P}=(1+\eta) \rho \mathrm{k}_{\mathrm{B}} \mathrm{T} / \mathrm{M}$. The ionization fraction $\eta$ is determined by a simultaneous solution of the Saha equation:

$$
\frac{\eta^{2}}{1-\eta}=2 \frac{u_{+}}{u_{0}} \frac{M}{\rho}\left(\frac{2 \pi m k_{B} T}{h^{2}}\right)^{\frac{3}{2}} e^{-\frac{I_{p}}{k_{B} T}},
$$

and the equation of state: 


$$
\rho e=\frac{\rho}{M}\left[\frac{(1+\eta) k_{B} T}{\gamma-1}+\eta I_{p}\right],
$$

where $I_{p}$ is the ionization potential, $u_{+}$and $u_{0}$ are the electronic partition functions ( 6 and 15, respectively, for silicon). This model is similar to the $1-\mathrm{D}$ models of Vertes and coworkers, ${ }^{10,11}$ which are based on a set of equations adopted by Zel'dovich and Raizer ${ }^{12}$ for weakly ionized plasmas. The Rusanov scheme is again used to solve these equations. ${ }^{9}$

Our model also contains source terms for mass density and energy input denoted by $S_{\rho}$ and $S_{e}$, respectively, in the mass density and energy equations. These source terms allow us to start the calculations with a clean slate and input mass and energy into the transport model according to the results from calculations of laser-target interactions using the thermal model we developed for this purpose. ${ }^{4}$ The mass density source is then given as $S_{\rho}=n_{l i q} M v_{r s}$ and the energy source as $S_{e}=n_{l i q} M k_{B} T_{v} /(\gamma-1)$, with $v_{r s}$ the recession speed, $T_{V}$ the vaporization temperature, and $n_{\text {liq }}$ the liquid density. For the duration of the laser pulse, these terms provide a dynamic source of mass and energy into the system, as occurs experimentally.

This continuous input of mass and energy into the system during the laser pulse has a significant impact on the maximum plume expansion velocity. This is shown in Fig. 5 where results from 1-D gas dynamic calculations of silicon plume transport in near vacuum (very dilute silicon background with density of $10^{10} \mathrm{~cm}^{-3}$ and temperature of $293 \mathrm{~K}$ ) with the dynamic source effect are displayed. A constant source of vapor is specified for $6 \mathrm{~ns}$ with a temperature $T_{\mathrm{V}}=7000 \mathrm{~K}$, the target recession speed of $\mathrm{v}_{\mathrm{rs}}=10^{3} \mathrm{~cm} / \mathrm{s}$, liquid density of $5.01 \times 10^{22} \mathrm{~cm}^{-3}, \gamma=5 / 3$ and sound speed $c_{S}=1.85 \times 10^{5} \mathrm{~cm} / \mathrm{s}$. Calculations where the density and temperature profiles at the end of the laser pulse are taken as initial conditions and allowed to freely expand are also shown for comparison in Fig. 5. The results of Fig. 5 do not include plume ionization or laser light absorption. The time evolution of the pressure at the solid surface with the source effect and for free expansion 
are shown on the top, while the plume front position as a function of time in both of these cases is shown on the bottom.

It is clear from Fig. 5 that high pressure at the surface is maintained for a longer time due to continuous ablation for the duration of the laser pulse compared to the free expansion case where the pressure at the surface rapidly drops as $1 /$ t. As a result, the plume expands with a higher maximum velocity than in the free expansion case as also shown in Fig. 5 where the plume front speed is $\sim 1.4 \times 10^{6} \mathrm{~cm} / \mathrm{s}$ or $\sim 7.5 \mathrm{c}_{\mathrm{S}}$ with the dynamic source effect compared to $\sim 5.5 \times 10^{5} \mathrm{~cm} / \mathrm{s}$ or $\sim 2 \mathrm{c}_{\mathrm{S}} /(\gamma-1)$ as it should be in the case of free expansion. Our calculations also show that the maximum expansion velocity can be increased further when ionization is included. Partial ionization at the front results in increased energy channeled into directed motion and a maximum velocity, which is $40 \%$ higher than with dynamic source effect alone. More details on the dynamic source effect for plume transport in vacuum, including analytical expressions for the steady-state density profile and maximum front velocity, which agree quite well with the results of Fig. 5, are given in Ref. 3. Suffice it to say that the maximum plume velocities obtained with the dynamic source effect come closer to matching the velocities inferred from experimental measurements.

Laser ablation experiments have shown that plume propagation in background gas can lead to stopping of the ablated material. In some cases, the material can even move backward, and several reflected shocks within the plume are apparent. This is borne out by the results of a 1-D gas dynamics calculation with dynamic source effect and a silicon background gas pressure of $200 \mathrm{mT}$ Torr as shown in Fig. 6. The total density, pressure, and velocity profiles are displayed as a function of distance from the target at four different times in the calculation up to $500 \mu \mathrm{s}$. Snowplowing of the background gas at the leading edge (a); rarefaction of the plume (b); slowdown and turnaround of the plume peak, the peak between target and front, by the snowplowed and piled-up background gas at the leading edge (c); and the subsequent reflection of the plume peak from the target (d) lead to 
multiple shocks between target and front. This sequence of events provides a likely scenario for what is observed experimentally in $\mathrm{KrF}$ laser ablation of graphite in argon at a pressure of 300 Torr. $^{5}$

\section{SCATTERING MODELS OF PLUME TRANSPORT}

As mentioned earlier, time-of-flight measurements have shown that there are two energetic components in the flux detected by ion probes when the laser-ablated plume expands in a background gas. This phenomenon is commonly referred to as plume splitting. Typical experimental fluxes are displayed in Fig. 7 for a silicon plume in argon and in helium with increasing pressure. It turns out that splitting of the total density and flux can be achieved in 1-D gas dynamics calculations. Results from such a calculation for silicon plume expansion in a 200 mTorr (silicon) background and in vacuum, where background, plume, and total densities and fluxes have been monitored separately, are shown in Fig. 8. The total density in Fig. 8(c) exhibits two peaks. The leading density peak is composed of snowplowed background gas, while the lagging peak is made up of the slowed down plume compared to what happens in vacuum [Fig. 8(a)]. Similarly, the fluxes displayed in Figs. 8(b) and 8(d) indicate that the background component reaches the detector first at plume vacuum speed, with the slowed down plume arriving later, which gives rise to the two peaks seen in the total flux.

However, more elaborate experimental diagnostics, such as emission and absorption spectroscopy, have shown that it is the plume itself that splits once a critical background gas pressure is reached. ${ }^{6}$ This has led us to consider scattering approaches that are more appropriate to longer mean free path situations at low background gas pressures than the short mean free path description afforded by the hydrodynamics and gas dynamics models applied so far to laser ablation modeling. 
Our scattering model is similar to that proposed by Koopman and Goforth, ${ }^{14}$ except that we do not rely on a fully ionized layer of background gas to scatter the plume ions through ion-ion collisions. This is because experimental confirmation of such an ionized layer of background gas has been elusive at best. Our scattering model includes plumeplume, plume-background, background-plume, and background-background collisional interactions with cross sections for elastic collisions that depend on the difference of the velocities of the interacting species to various inverse powers. The calculated fluxes at a distance of $2 \mathrm{~cm}$ from the target are displayed in Fig. 9 for a silicon plume propagating into argon at a pressure of 200 mTorr. They indicate that part of the silicon plume gets to the detector at vacuum speed. Splitting of the silicon plume itself into two energetic components is also seen in Fig. 9. Extensions of this promising scattering model, as well as application of the Direct Simulation Monte Carlo techniques found in Bird, 15 are currently under way.

\section{SUMMARY}

Several key physics issues at the core of the laser ablation process have been addressed with our models of plume transport. We have shown that the gross hydrodynamic features of plume expansion in vacuum and in background gas, as exemplified by emission patterns imaged with an ICCD camera, are well reproduced by the particle-in-cell hydrodynamics and continuum gas dynamics models. We have identified a dynamic source effect with our gas dynamics models, which results from continuous input of mass and energy during the laser pulse. This dynamic source effect yields higher plume velocities than conventional free expansion models that come closer to matching experimentally measured plume speeds. We have also presented preliminary results from a scattering approach to plume transport, which shows promise in modeling the two energetic components experimentally detected in time-of-flight measurements. 


\section{ACKNOWLEDGMENTS}

This research was sponsored at the Oak Ridge National Laboratory (ORNL) by the Laboratory Directed Research and Development (LDRD) Program and by the Division of Materials Sciences, U. S. Department of Energy, under contract DE-AC05-84OR21400 with Lockheed Martin Energy Systems, Inc. K. R. Chen and Chun-Li Liu were supported by an appointment to the ORNL Research Associate Program administered jointly by the Oak Ridge Institute for Science and Education (ORISE) and ORNL.

\section{DISCLAIMER}

This report was prepared as an account of work sponsored by an agency of the United States Government. Neither the United States Government nor any agency thereof, nor any of their employees, makes any warranty, express or implied, or assumes any legal liability or responsibility for the accuracy, completeness, or usefulness of any information, apparatus, product, or process disclosed, or represents that its use would not infringe privately owned rights. Reference herein to any specific commercial product, process, or service by trade name, trademark, manufacturer, or otherwise does not necessarily constitute or imply its endorsement, recommendation, or favoring by the United States Government or any agency thereof. The views and opinions of authors expressed herein do not necessarily state or reflect those of the United States Government or any agency thereof. 


\section{References}

1. K. L. Saenger, in Pulsed Laser Deposition of Thin Films, edited by D. B. Chrisey and G. K. Hubler (Wiley, New York, 1994) pp. 581-604.

2. J. T. Cheung, in Pulsed Laser Deposition of Thin Films, edited by D. B. Chrisey and G. K. Hubler (Wiley, New York, 1994) pp. 1-22.

3. R. M. Gilgenbach, C. H. Ching, J. S. Lash, and R. A. Lindley, Phys. Plasmas 1, 1619 (1994).

4. J. N. Leboeuf, K. R. Chen, J. M. Donato, D. B. Geohegan, C. L. Liu, A. A. Puretzky, and R. F. Wood, "Dynamical modeling of laser ablation processes," in Film Synthesis and Growth Using Energetic Beams, edited by H. A. Atwater, J. T. Dickinson, D. H. Lowndes, A. Polman (Mater. Res. Soc. Proc. 388, Q1.1, San Francisco, CA, 1995) to be published.

5. A. A. Puretzky, D. B. Geohegan, R. E. Haufler, R. L. Hettich, X.-Y. Zheng, and R. N. Compton, in Laser Ablation: Mechanisms and Applications II, edited by J. C. Miller and D. B. Geohegan (AIP Conference Proceedings 288, American Institute of Physics, New York, 1994) pp. 365-374 .

6. D. B. Geohegan and A. A. Puretzky, Appl. Phys. Lett. 67, 197 (1995).

7. J. N. Leboeuf, T. Tajima, and J. M. Dawson, Journ. Comput. Phys 31, 379 (1979).

8. F. Kazeminejad, J. N. Leboeuf, F. Brunel, and J. M. Dawson, Journ. Comput. Phys. 104, 398 (1993).

9. G. A. Sod, Journ. Comput. Phys 27, 1 (1978).

10. A. Vertes, P. Juhasz, M. D. Wolf, and R. Gijbels, Scanning Microscopy 2, 1853 (1988).

11. L. Balazs, R. Gijbels, and A. Vertes, Analytical Chemistry 63, 314 (1991). 
12. Ya. B. Zel'dovich and Yu. P. Raizer, Physics of Shock Waves and HighTemperature Hydrodynamic Phenomena (Academic Press, New York and London, 1966).

13. K. R. Chen, J. N. Leboeuf, R. F. Wood, D. B. Geohegan, J. M. Donato, C. L. Liu, and A. A. Puretzky, "Accelerated expansion of laser-ablated materials near solid surface," Phys. Rev. Letts., to be published.

14. D. W. Koopman and R. R. Goforth, Phys. Fluids 17, 1560 (1974).

15. G. A. Bird, Molecular Gas Dynamics and the Direct Simulation of Gas Flows (Clarendon Press, Oxford, 1994). 


\section{Figure Captions}

Fig. 1 Results from 2-D particle-in-cell hydrodynamics calculations of plume expansion in vacuum and in background gas: $x-y$ space projection of plume particles in vacuum (top), of plume and background particles (middle), of background particles alone (bottom) as a function of time.

Fig. 2 Results from 2-D particle-in-cell hydrodynamics calculations: $x-y$ space projection of plume and background particles at the same time but for increasing values of the viscous drag coefficient $v$, such as (a) $v=0$, (b) $v=10^{-2}$, and (c) $v=1.0$.

Fig. 3 Results from 2-D gas dynamics calculations of plume expansion in vacuum (top) and in background gas (bottom): contours of total density (plume and background) as a function of time.

Fig. 4 Results from 2-D gas dynamics calculations of expansion of two plumes in vacuum: contours of density as a function of time.

Fig. 5 Results from 1-D gas dynamics calculations with dynamic source effect and for free expansion: (a) pressures at target surface as a function of time and (b) positions of the leading edge of the plume as a function of time.

Fig. 6 Results from 1-D gas dynamics calculation with dynamic source effect and 200 mTorr background gas: profiles of total density, pressure, and velocity as a function of position and at time (a) $10 \mathrm{~ns}$, (b) $1 \mu \mathrm{s}$, (c) $100 \mu \mathrm{s}$, and (d) $500 \mu \mathrm{s}$. 
Fig. 7 Ion probe signals measured at $5 \mathrm{~cm}$ from the target for laser ablation of silicon in (a) argon and (b) helium at different pressures.

Fig. 8 Results from 1-D gas dynamics calculation in the case of free expansion: (a) plume density profile as a function of distance from the target in vacuum; (b) plume flux (density $\times$ velocity) in vacuum at $5 \mathrm{~cm}$ as a function of time; (c) plume, background, and total density profiles; and (d) plume, background, and total fluxes at $5 \mathrm{~cm}$ as a function of time.

Fig. 9 Result from 1-D scattering model: calculated fluxes at $2 \mathrm{~cm}$ for silicon plume expansion in $200 \mathrm{mTorr}$ of argon 

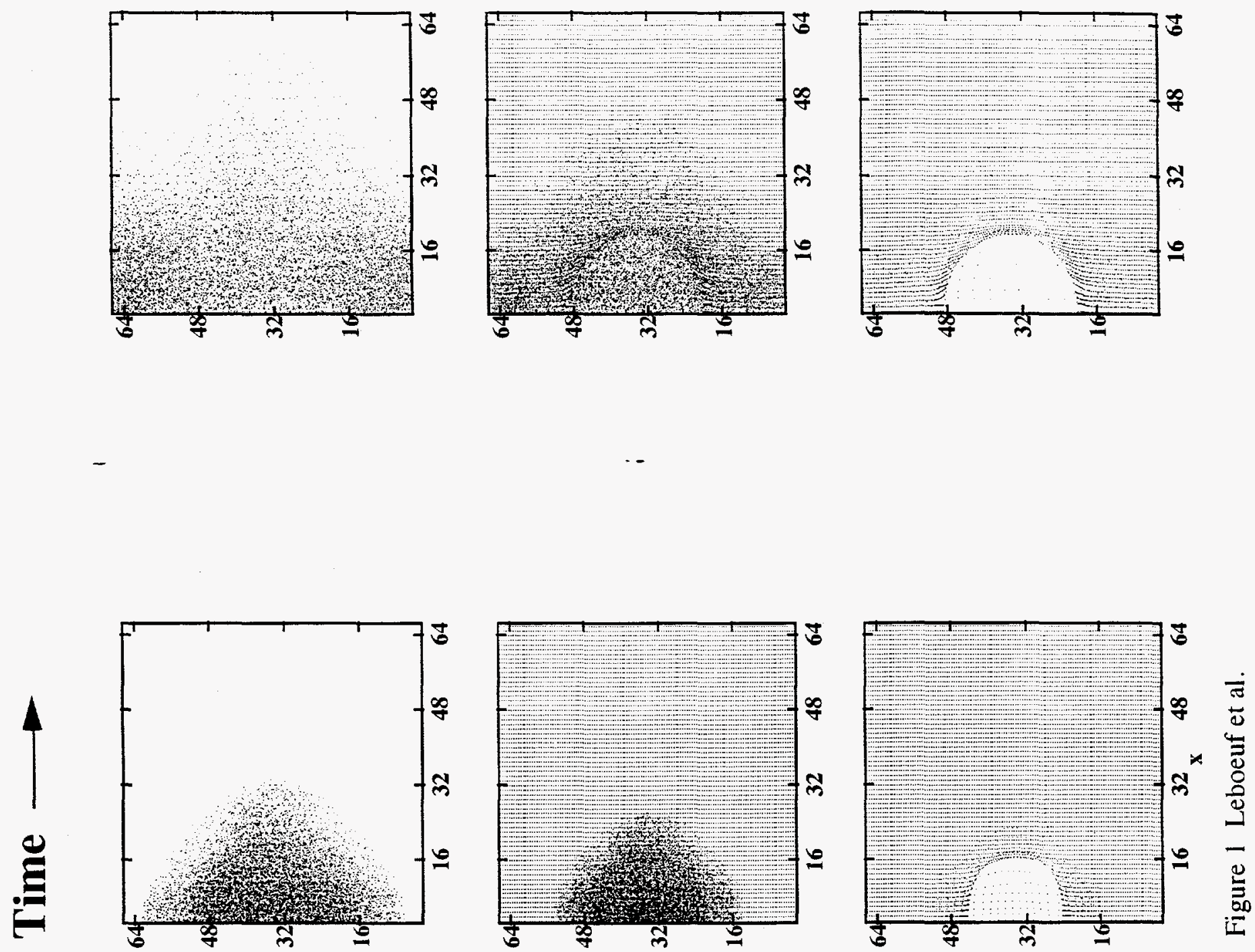

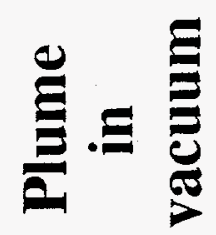
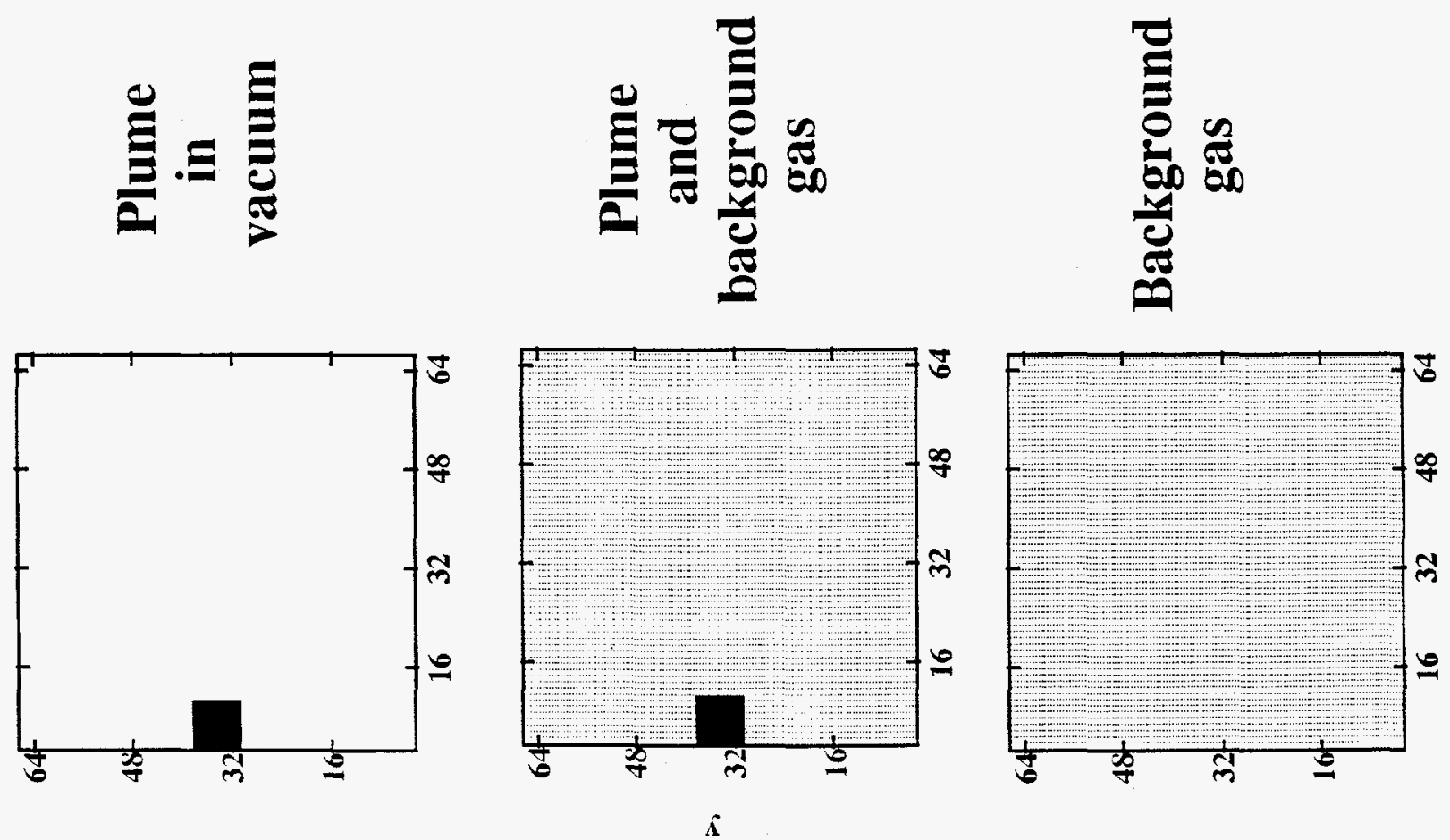
a)

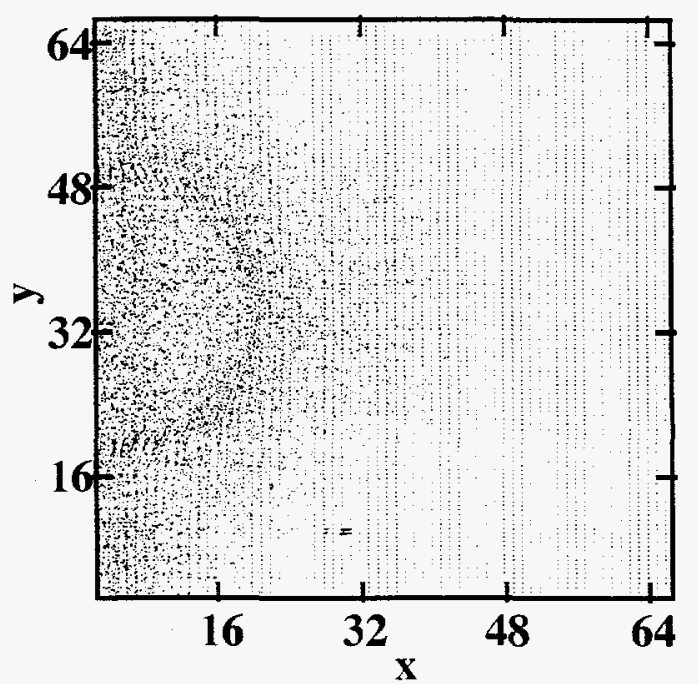

b)

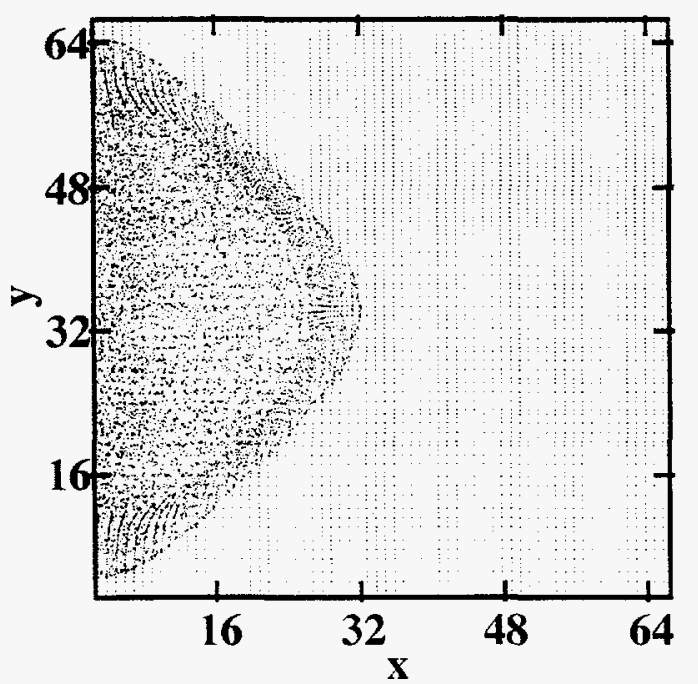

c)

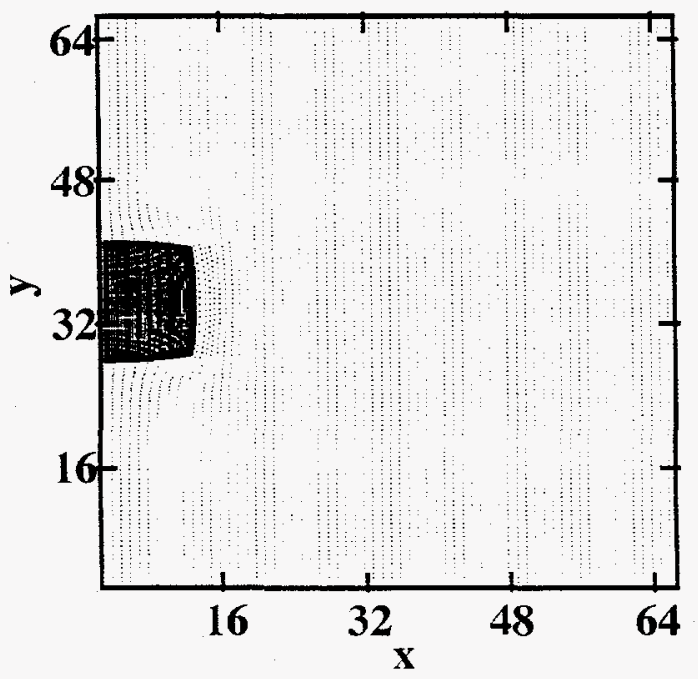

Figure 2 Leboeuf et al. 

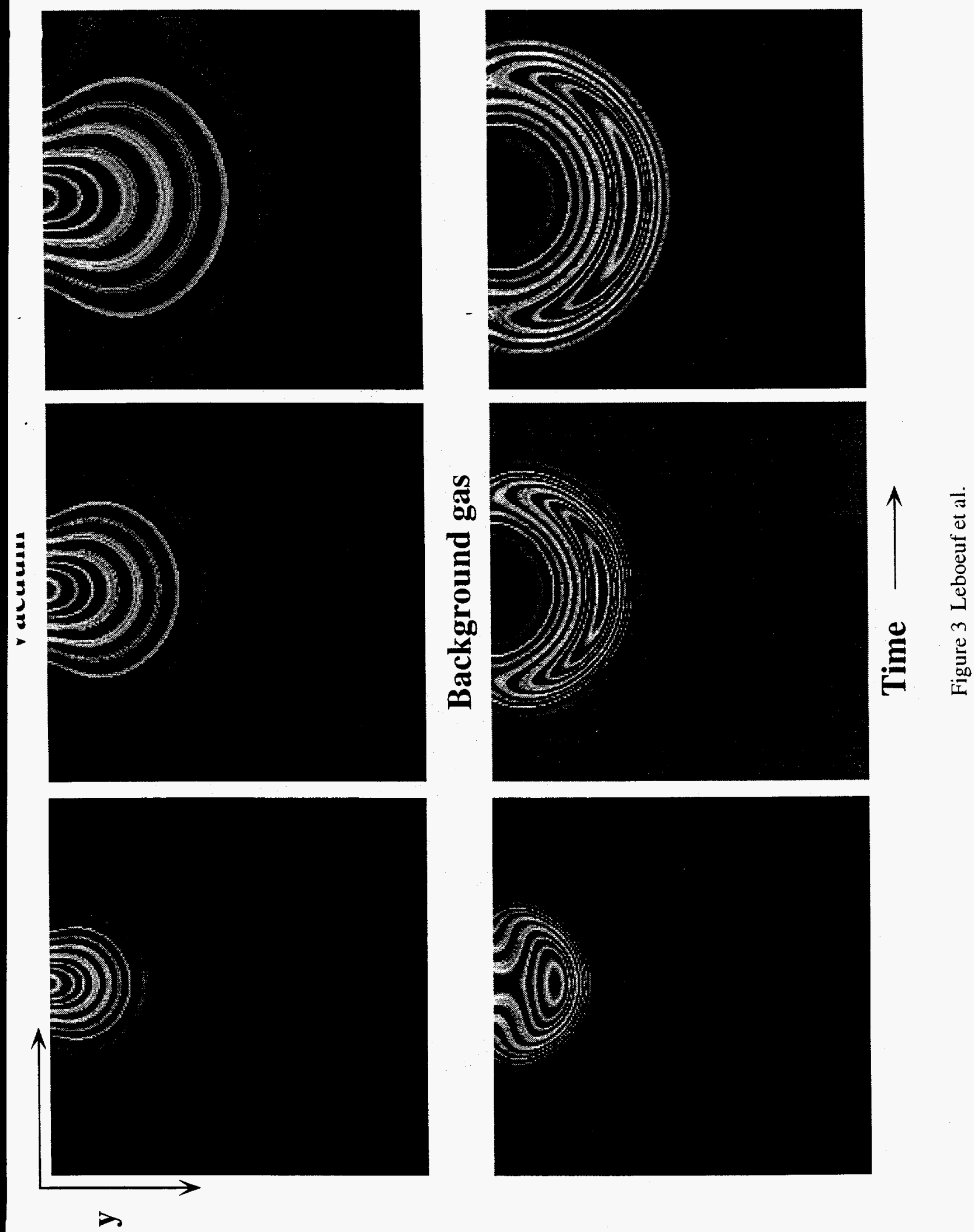

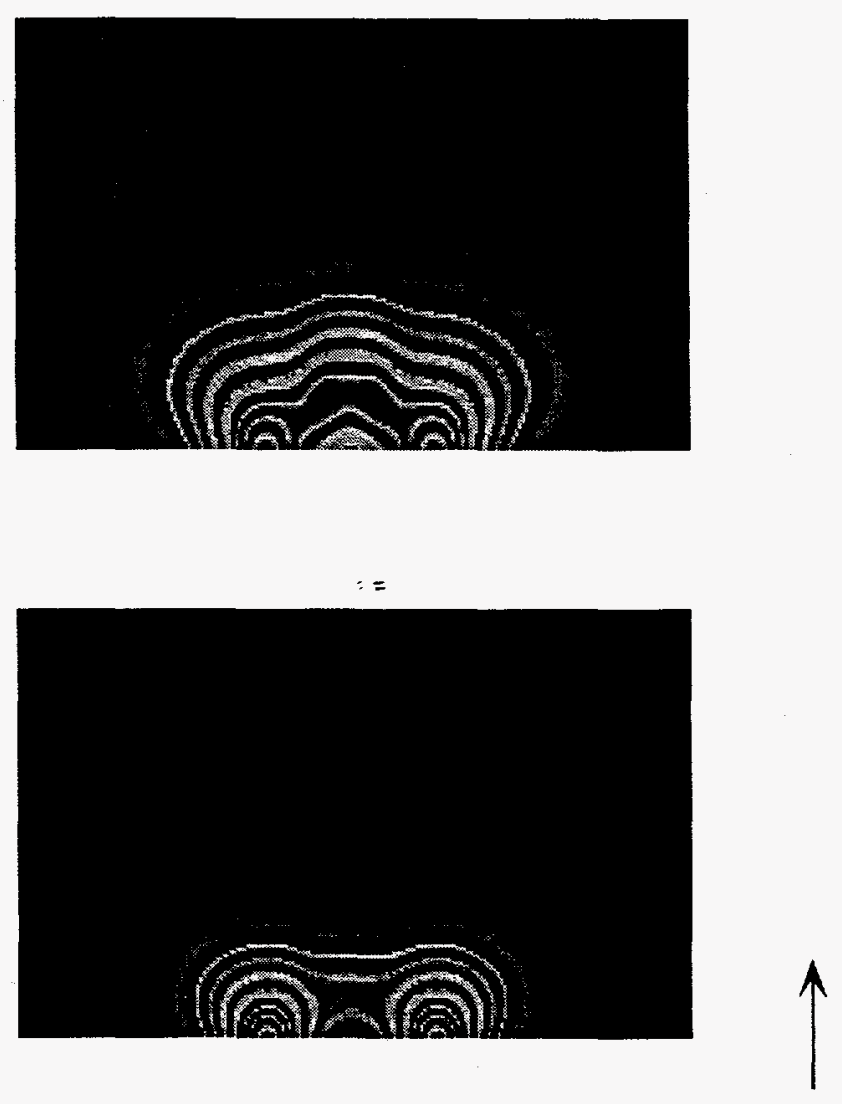

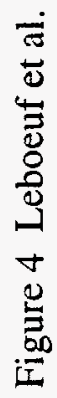

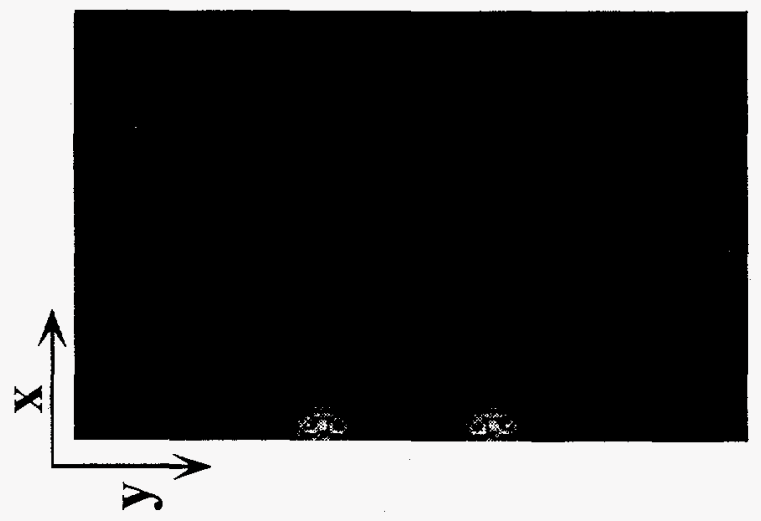



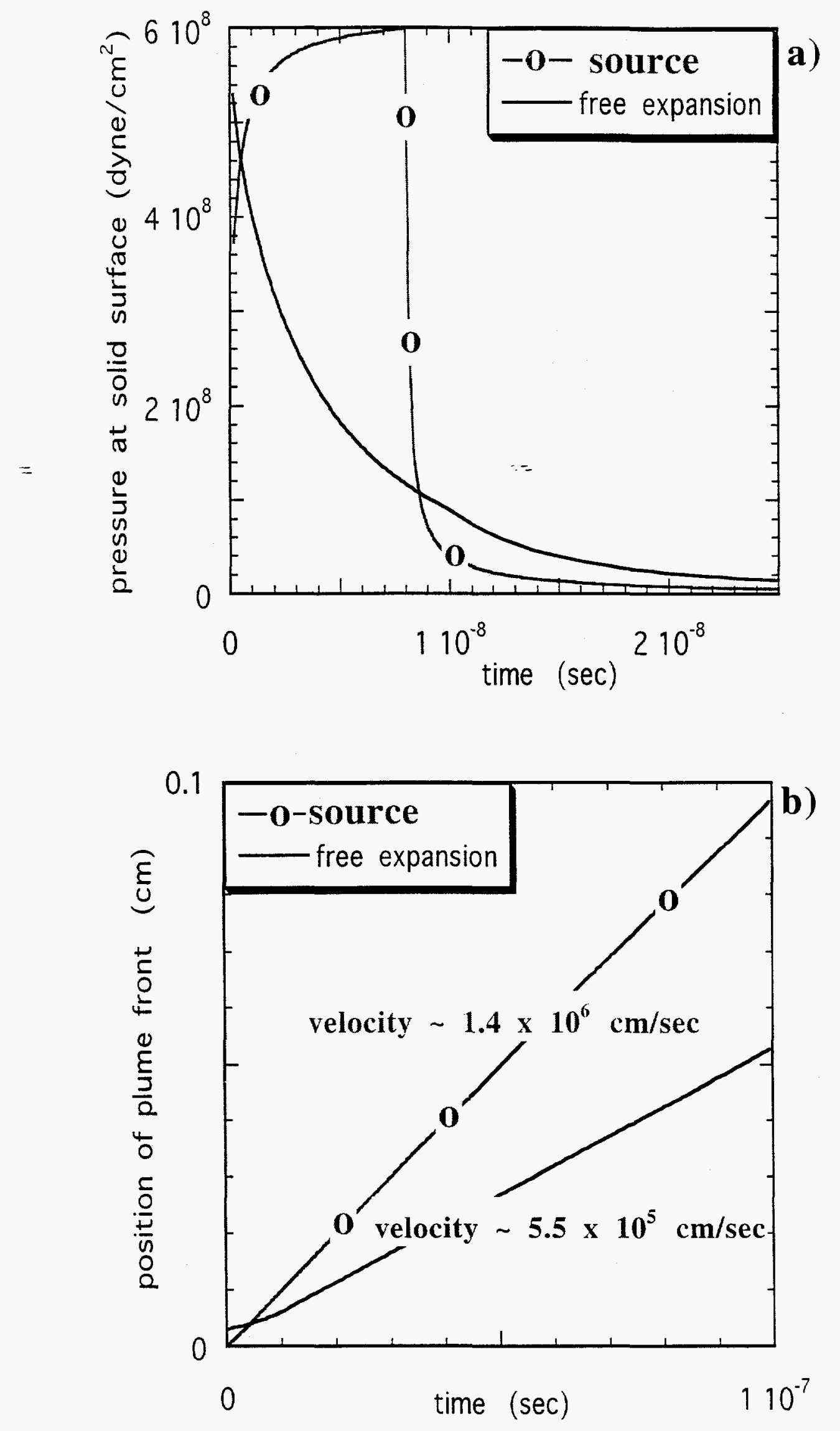

Figure 5 Leboeuf et al. 
(a)

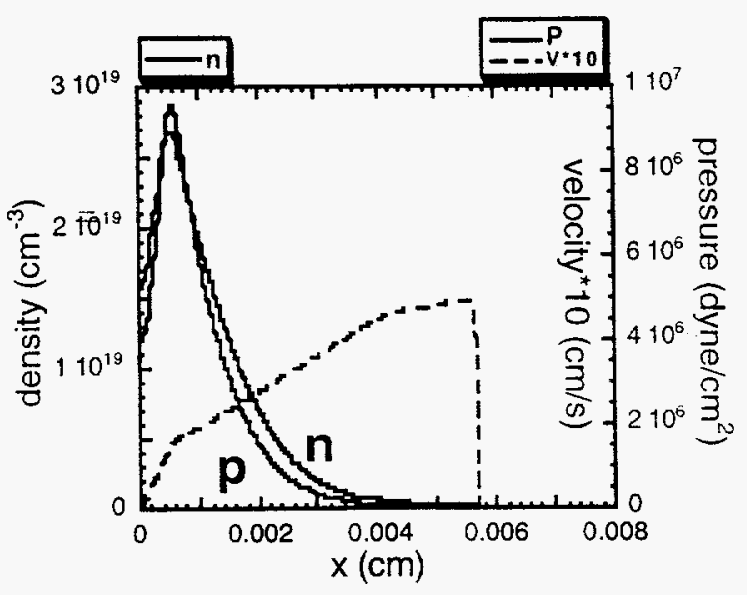

(c)

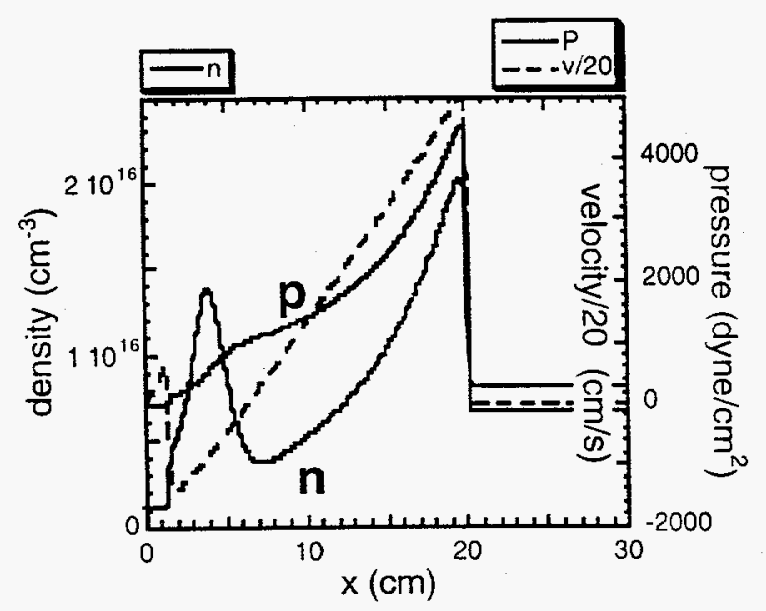

(b)

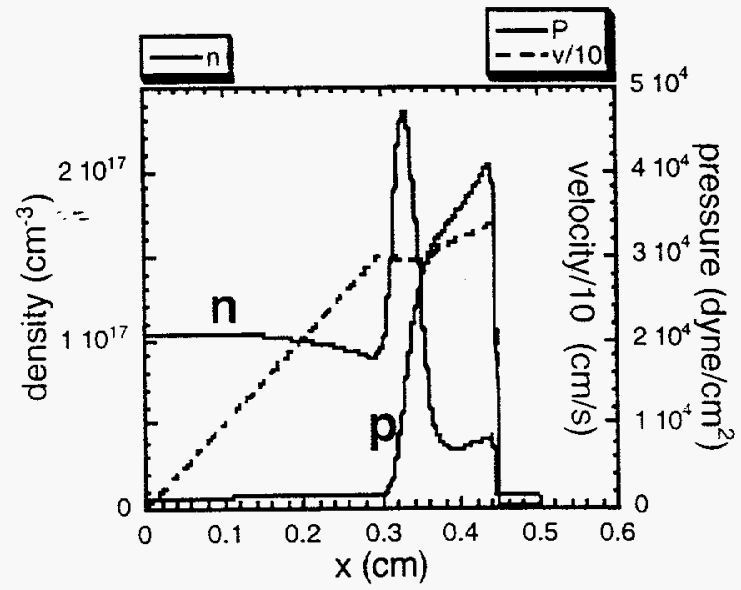

(d)

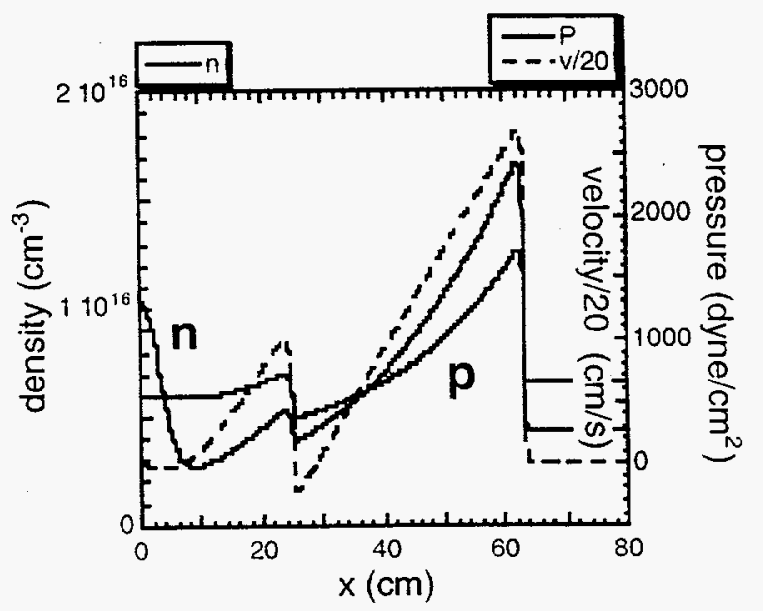

Figure 6 Leboeuf et al. 
a)

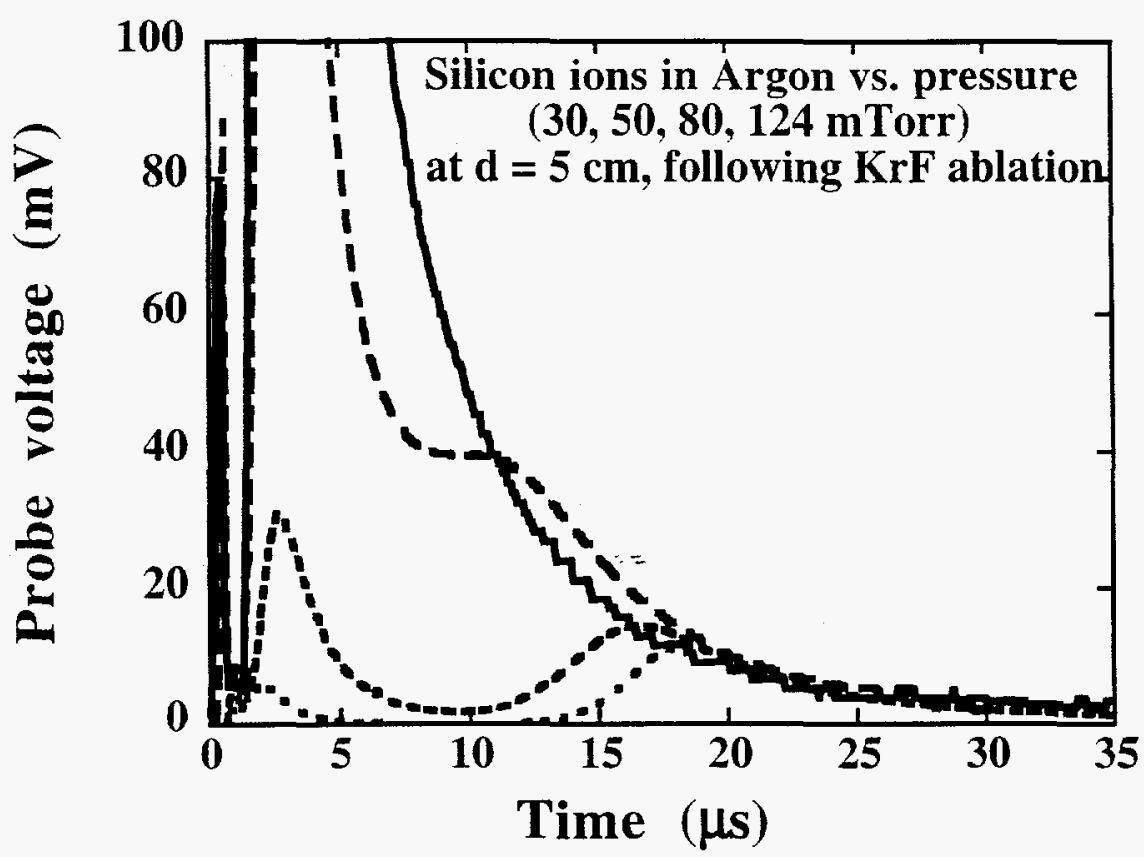

b)

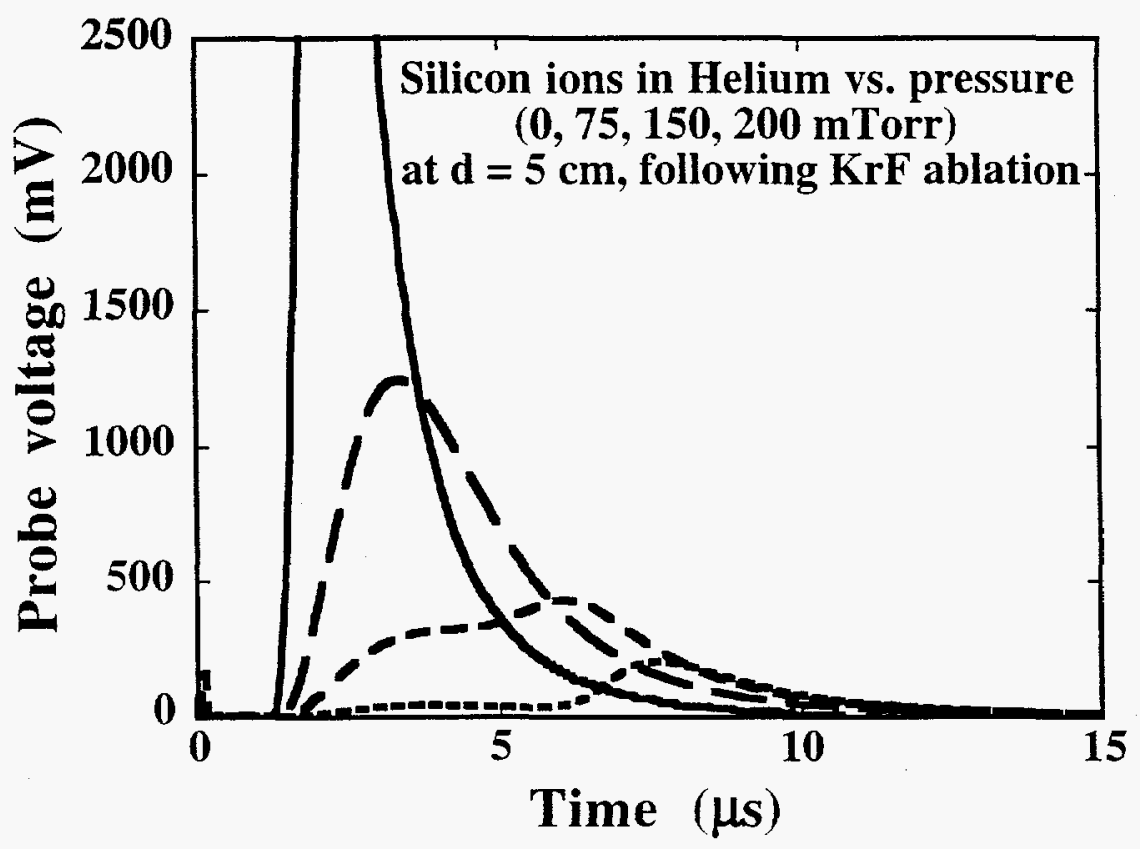

Figure 7 Leboeuf et al. 


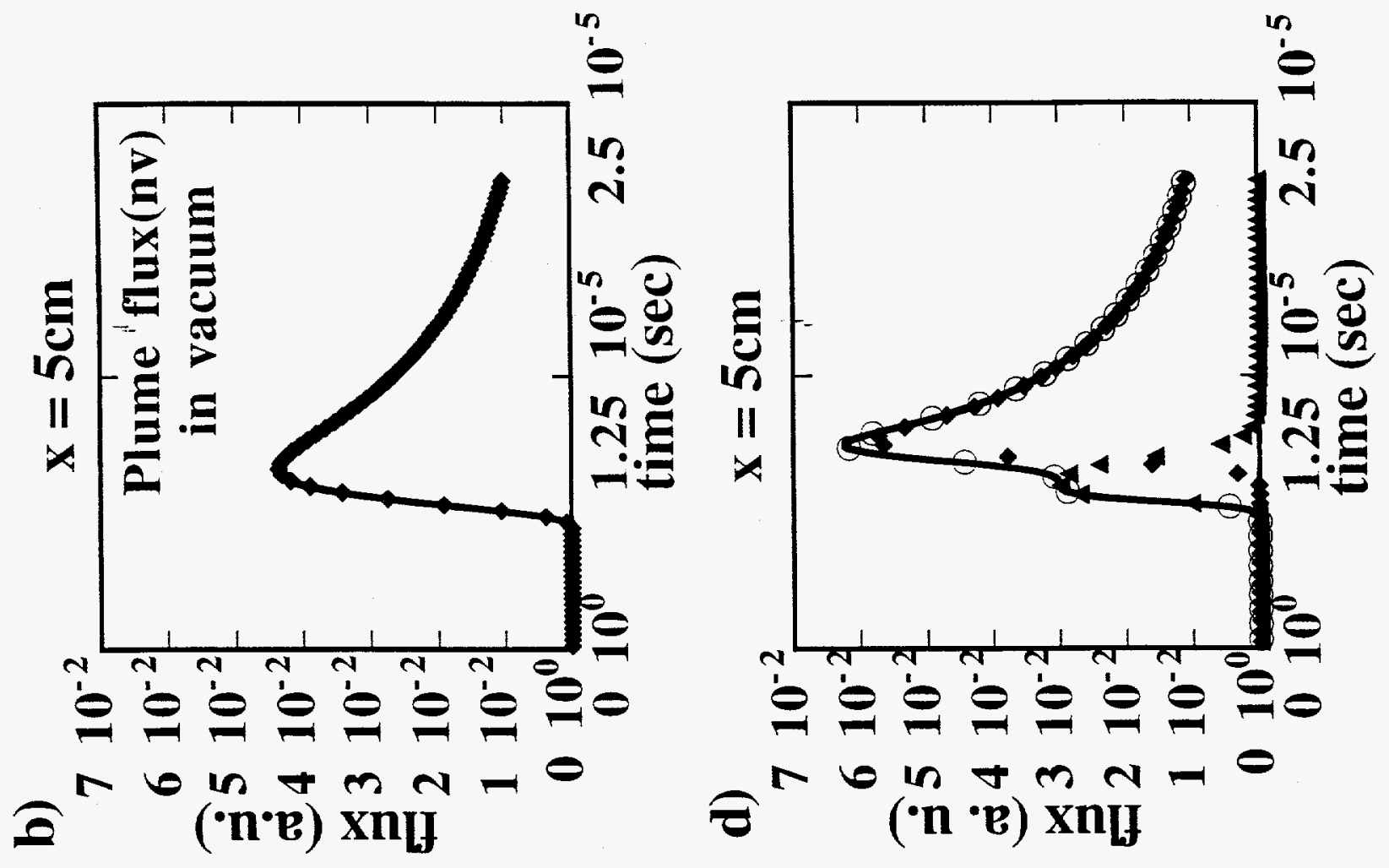

क
0
4
0
0
0
0
0
0
0
0
01

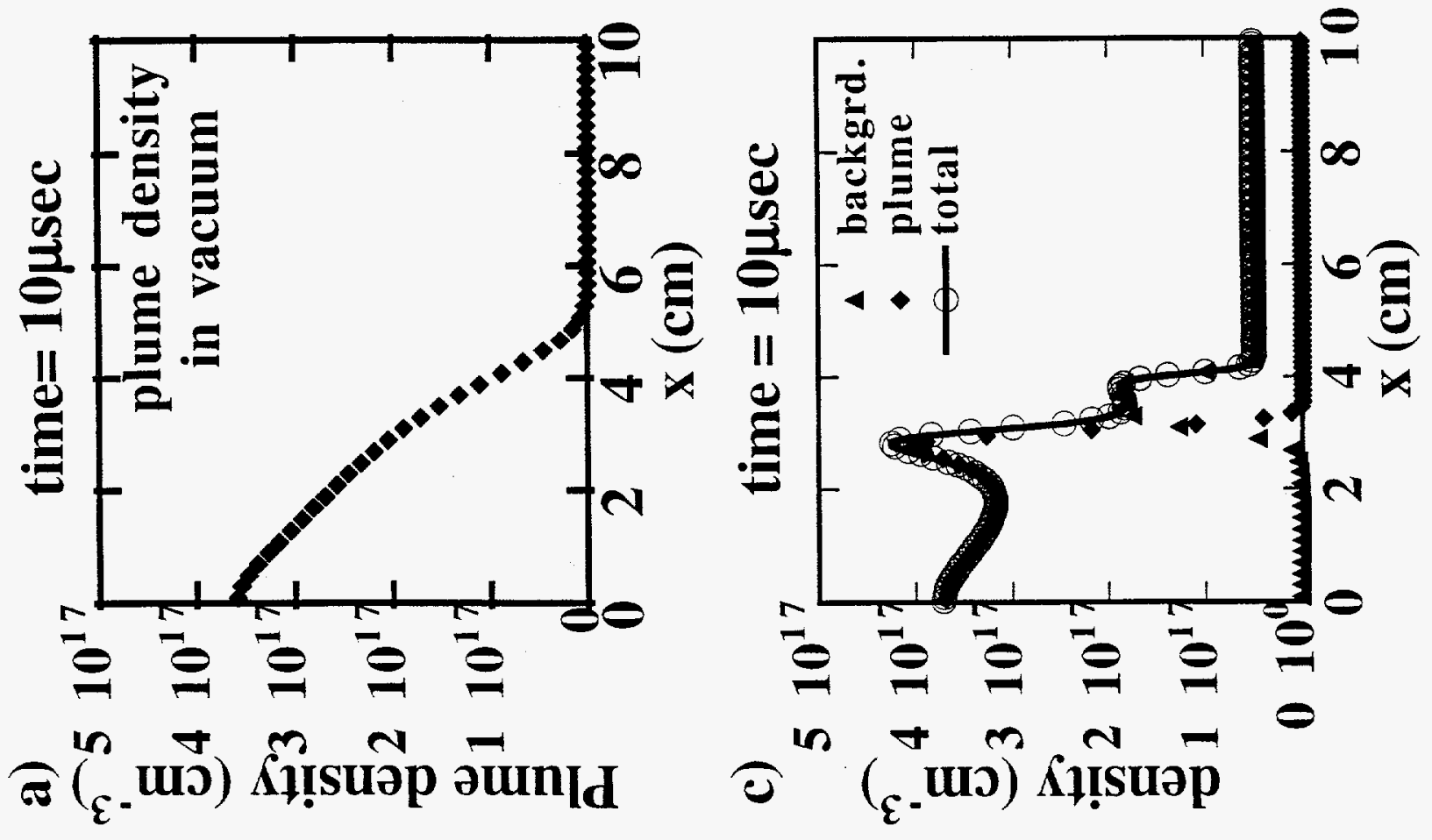




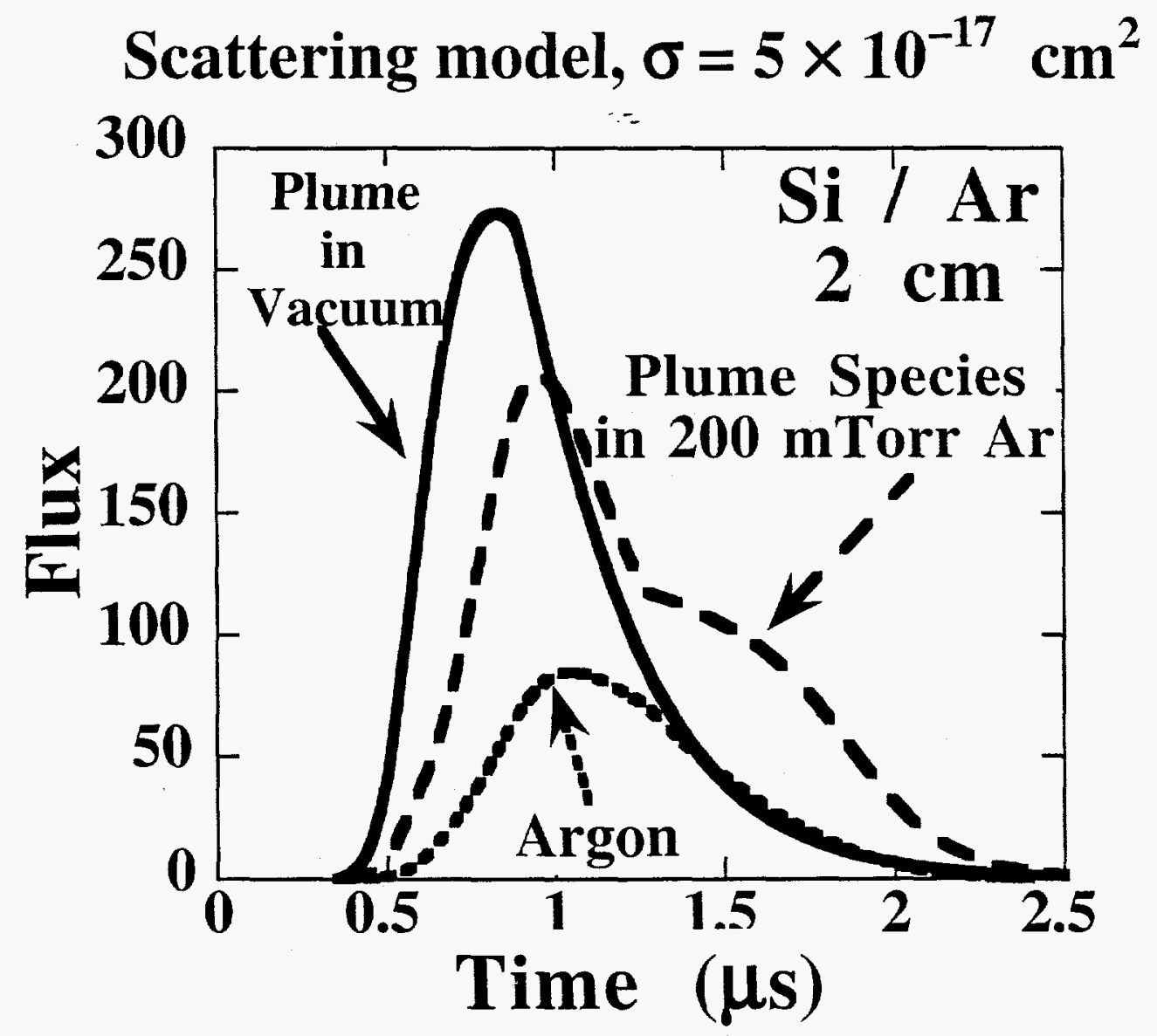

Figure 9 Leboeuf et al. 